\title{
Controlling Proteome Degradation in Daphnia pulex
}

\author{
Catherine Jarrett Kemp ${ }^{1,{ }^{*}}$ and Dietmar Kültz ${ }^{1}$ \\ ${ }^{1}$ Department of Animal Science, University of California, Davis, California 95616
}

\begin{abstract}
An attempted proteomic study of Daphnia pulex response to sodium chloride exposure revealed an unexpected result: very strong proteome degradation previously observed in D. pulex is halted when animals have been exposed to elevated salinity prior to protein extraction. Further characterization of conditions controlling proteome degradation shows that the proteolytic factor(s) is/are present in an active form under chemically denaturing conditions in extract from control "normal/healthy" animals, and can be negatively regulated by prior in vivo salinity exposure of animals or inhibited by post-homogenization heating of samples. We interpret these data as evidence for salinity-induced inhibition of proteolysis in Daphnia.
\end{abstract}

\section{Introduction}

The recent annotation of the Daphnia pulex genome has drawn attention to an ongoing effort to establish this organism as a research model for ecological genetics, a field devoted to studying the interplay of the environment with genomes of organisms. D. pulex has many useful attributes, including a short lifecycle, rapid clonal reproduction alternating with inducible sexual reproduction, wide geographic distribution, and local adaptations to a wide range of environmental conditions. These advantages have inspired a concerted research effort spearheaded by the Daphnia Genomics Consortium to sequence and develop research tools for studying this organism (Colbourne et al., 2011). Given the extensive investment from the research community in developing $D$. pulex into a model for ecological genetics, it is critical that researchers be able to reliably study all classes of biomolecules in order to fully characterize organismal response to the environment and facilitate future systems biology approaches. However, attempts to study the D. pulex proteome have proved frustrating due to rapid and extensive protein degradation.

Recently published two-dimensional gel electrophoresis (2DGE) proteomic studies of $D$. pulex have revealed major protein degradation and note that no assayed commercially available protease inhibitors prevented this breakdown (Schwerin et al., 2009; Zeis et al., 2009; Frohlich et al., 2009). However, Frohlich et al. (2009) noted that a stable onedimensional (1D) gel can be obtained, suggesting that degradation control is possible and proteolysis likely occurs post-homogenization rather than as the natural state within the intact organism. The implications of rapid whole-proteome degradation go beyond hindering reliable proteomics approaches. Any method assaying proteins in Daphnia homogenates potentially introduces considerable error as the precise rate, nature, and control of the unusually high protein breakdown in Daphnia samples is currently unknown. Furthermore, rapid and massive protein degradation seems to be the 'default' state in homogenized untreated animals in standard buffers, which presents a major and significant variable in protein-based studies. Therefore, it is essential to devise methods that minimize proteome

"Correspondence to: Catherine Jarrett Kemp, Department of Animal Science, One Shields Avenue, Davis CA 95616 (tel: (530)752-7322 fax: (530)752-0175, cakemp@ucdavis.edu). 
degradation in D. pulex and to understand the conditions that lead to it, enabling control of this undesirable feature in an otherwise highly useful model organism.

\section{Materials and Methods}

\section{Animal Maintenance}

D. pulex (the "chosen one" strain obtained from Celia Chen, Dartmouth College) were cultured in ten gallon fish tanks in artificial Daphnia medium (Refardt and Ebert, 2007), 20 C, 16L:8D photoperiod, and fed $1 \mathrm{ml}$ commercial Nannochloropsis (Reed Mariculture) three times weekly per $20 \mathrm{~L}$ culture media.

\section{Animal Usage}

One day prior to exposure, cultures were pooled and the medium decanted to remove accumulated debris. Immediately prior to exposures, all animals were pooled in a mesh bag and rinsed to retain only adults and remove neonates via size exclusion in order to prevent bias within samples. Adults and neonates are believed to have different osmoregulatory systems (Bianchini and Wood, 2008), and in preliminary studies the salinity levels used in these experiments stopped reproduction (data not shown). Animals were then randomly distributed among $2 \mathrm{~L}$ beakers containing either control $(15 \mathrm{mOsm})$ or saline $(150 \mathrm{mOsm})$ artificial media; salinity was increased by adding $\mathrm{NaCl}$ and measured using a model 3300 Advanced Micro Osmometer (Advanced Instruments Inc). At the end of the exposure period, the animals were collected in a fine mesh-bottomed cup and roughly 200/sample transferred to a $2 \mathrm{ml}$ low-retention microcentrifuge tube. Excess media was immediately removed using a $1 \mathrm{ml}$ pipette and samples were snap-frozen in liquid nitrogen, then transferred to $-80^{\circ} \mathrm{C}$ for storage.

\section{Molecular Methods}

Sample homogenizing and SDS-PAGE (1D and 2D) were carried out as described (Dowd et al., 2010) with the following modification: for $2 \mathrm{D}$ experiments ( $\mathrm{n}=6$ per treatment), samples were prepared using a phenol:chloroform:isoamyl (PCIA) method (Antonioli et al., 2009). The PCIA method was used in place of acetone precipitation as preliminary optimization studies demonstrated a $\sim 2.5 \times$ increase in spot visibility (data not shown).

\section{Protease inhibitor screening}

Individual protease inhibitors (Calbiochem Protease Inhibitor Set catalog \#539128) E-64, EST(E64-D), Leupeptin, Pepstatin A, TLCK(HCL), TPCK, Complete Minitab protease inhibitor cocktail (Roche), or no inhibitor control were added to Radio-ImmunoPrecipitation Assay (RIPA) homogenization buffer prior to manual disruption at maximal effective concentration. Homogenates were either immediately electrophoresed or left at room temperature for 6 hours prior to separation via 1D SDS-PAGE, and each sample was run both with and without pre-load heating.

\section{Azocasein assay}

This assay was carried out in duplicate exactly as described in protocol SSAZOC03 supplied with azocasein substrate (Sigma Aldrich), except identical reactions were terminated after 10,30 , or 60 minutes. The absorbance values at $440 \mathrm{~nm}$ were adjusted for blank and total protein concentration, then averaged and plotted against time.

\section{Gel image quantification}

Protein staining in 1D gels was quantified using ImageQuant 5.1 (Molecular Dynamics). Each lane was divided at the $37 \mathrm{kD}$ marker into an upper and lower quantification box; total 
volume data was exported to Excel for analysis by dividing top volume/bottom volume to generate a ratio which reflected the relative amount of protein found in the upper vs. lower portion of the gel.

\section{Results and Discussion}

\section{In vivo sodium chloride exposure prevents proteome degradation}

An initial attempt at typical 2DGE analysis comparing control and sodium chloride-exposed animals yielded an unexpected result: post-extraction degradation of proteome samples was prevented in salinity treated animals (Fig. 1A, B). Replicate gels within control or salinity groups were very similar and completely overlayable; a typical representation of the data is shown (Fig. 1A, B). However, quantitative comparison of control and salinity gels proved impossible as very little of the proteome map could be matched for quantitative analysis (Fig. 1D). In the control gels spots occurred almost exclusively in the lower left quadrant (low molecular weight, acidic) with a high degree of streaking and background 'smudge', indicating protein breakdown (Fig. 1A). In contrast, spots on salinity gels were wellresolved and covered the full range of molecular weight and isoelectric point (Fig. 1B). We conclude that salinity exposure leads to endogenous regulation of the proteolytic factor(s) present in D. pulex, and hypothesize that one or more proteases are responsible for this effect. Proteases are the most likely factor responsible given that most other molecules (e.g., catalytic RNAs) should have been removed during the PCIA protein purification.

To further assess degradation rates, we performed an azocasein activity assay and found that proteolytic activity is entirely eliminated within salinity homogenates (Fig. 2A). This quantitative assay confirms the qualitative observation of salinity-induced degradation control, presented in Fig. 1.

\section{Degradation occurs in chemically denaturing conditions}

Because it seems counterintuitive and highly unlikely that constitutive whole-proteome degradation could be tolerated within living animals, we hypothesized that the proteome breakdown occurred post-homogenization during sample processing as one or more proteolytic factors were released from a confined cell compartment, resulting in protein degradation as is often observed (North and Beynon, 2001). Our data further suggest that the proteolytic factor(s) is inactivated by high salinity in vivo, thus minimizing proteome degradation during sample preparation. In searching for ways to inhibit the proteolytic factor(s) in vitro in extracts from control samples we observed degradation in a 1D SDSPAGE gel visible after the transition to $8 \mathrm{M}$ urea-containing buffer, and not during the initial homogenization step (Fig. 3). One-dimensional banding patterns were intact in RIPA homogenization buffer, and samples in this buffer left at room temperature for six hours prior to electrophoresis showed no additional visible degradation (data not shown).

To ensure that degradation was not method-specific, we compared PCIA-prepared samples to the traditional $10 \%$ TCA/ $90 \%$ acetone method and observed the same overall pattern of degradation, except that PCIA resulted in a much higher spot yield of breakdown products. To further confirm this result, we ground frozen tissue in liquid nitrogen and suspended this homogenate directly in urea buffer, and again obtained the degraded 2D pattern (data not shown). We additionally found that the standard step of adding SDS prior to loading 1D gels, when not followed immediately by heating, also led to degradation (Fig. 3B, 4). Thus, the proteolytic effect occurred every time after a sample was exposed to chemically denaturing conditions (8M urea, SDS, $10 \%$ TCA), and was not specific to a particular processing method. 


\section{Salinity-induced cessation of degradation requires in vivo exposure for at least one hour}

Next, we considered whether the change in degradation could be due to differences in salt concentration during homogenizing and processing or if it reflected real biological events. If activity of the supposed proteolytic factor required lower saline concentrations during posthomogenization processing, then salt-exposed samples might simply have a higher osmolality due to small amounts of media carried over during sample collection, or due to a higher salt load within the organism from acute salinity exposure. On average, salinityexposed samples inhomogenization buffer did have a marginally higher osmolality (314.7 \pm 1 mOsm control; $328 \pm 0.3 \mathrm{mOsm}$ salt). However, if this slight osmolality change in the homogenization bufferwere sufficient to induce alteration of proteolytic activity, then all salt-exposed samples would be expected to exhibit the same degradation pattern. This prediction is inconsistent with our actual data, which indicate that sixty minutes of in vivo salinity exposure is necessary for negative regulation of the proteolytic factor(s) to occur (Fig. 3). Earlier salinity-exposed time points ( $\mathrm{t}=5-30$ minutes) still retained degradation activity like that of non-exposed controls. We also homogenized control samples in RIPA with added $\mathrm{NaCl}$ so that the overall osmolality of the buffer was equal to (330mOsm) or higher $(613 \mathrm{mOsm})$ than that of salinity samples, without any change in the degradation pattern of control samples, indicating that simple carryover of excess salt alone cannot explain our observations (data not shown). The very small differences in sample type osmolality, requirement for a full hour of salinity exposure, and inability to control degradation by adding $\mathrm{NaCl}$ during processing all suggest real biological regulation controls activity of the proteolytic factor(s) present in $D$. pulex.

\section{Mode of regulation following salinity exposure}

To test potential mode of regulation we mixed control and salinity homogenates in varying proportions and visualized these using native 1D SDS-PAGE (fig. 5). Degradation is visible in control and all mixed samples; only pure salinity homogenate shows no evidence of proteolytic activity. To ensure that the apparent degradation was not simply dilution of the stable proteome contribution from salinity samples, we quantified the gel by creating a ratio of protein staining intensity of top/bottom within each lane. This produced a ratio which reflects the relative amount of protein distributed in high (intact) vs. low (degraded) molecular weight regions of the gel; a higher ratio indicates more intact protein and thus less degradation. This analysis shows a positive exponential fit, suggesting that degradation capability is transferred from the control to salinity homogenate when mixed (fig. 5). Pure dilution would be expected to result in a linear fit and thus we reject the possibility that no additional degradation is occurring in the mixtures. If positive regulation of an inhibitor was occurring in the salinity samples, then we predict this inhibitor would also act to prevent degradation of the control homogenate within the mixture and result in a logarithmic fit (the opposite result of observed). Therefore, our data suggests that degradation ability is transferred from the control to the salinity samples, indicating that negative regulation of the proteolytic factor(s) is occurring in the salinity samples.

\section{Degradation is heat-sensitive}

Typical sample preparation for 1D gels includes a heating step immediately following the addition of loading buffer (for samples in homogenization buffer, but never urea due to cyanide formation and carbamylation (Kurien and Scofield, 2012)); however in an instance of running a 1D SDS-PAGE gel, we omitted this heating step and found that control samples were degraded while salinity samples retained clean banding patterns. Previously, no degradation had been observed in 1D gels. We hypothesized that a short heating step immediately following homogenizing would be sufficient to permanently inactivate the proteolytic factor(s) even after transfer to denaturing conditions and would reproduce the effects of salinity treatment. Heated control samples $\left(5 \mathrm{~min}, 95^{\circ} \mathrm{C}\right)$ generated a $2 \mathrm{D}$ map 
comparable to that of salinity-treated animals (Fig. 1). However, these two treatments (in vivo salinity and in vitro heat) did not result in completely identical 2D gels; differences observed are most likely the result of true in vivo protein regulation in response to salinity stress.

Further experiments demonstrate that $70^{\circ} \mathrm{C}$ is the minimal temperature required for complete inactivation during a 10 minute heating, and 2 minutes is the minimal time required for complete inactivation while at $95^{\circ} \mathrm{C}$ (data not shown). Use of these milder conditions should aid in avoiding problems associated with protein modification due to high temperature (Kurien and Scofield, 2012) and inclusion of this simple heating step in 2D sample preparation offers one method to address the degradation problems associated with high proteolytic activity in Daphnia extracts, as long as the sample is intended for non-native assays. Furthermore, the similarity between heated control samples and saline samples further supports our initial hypothesis that the drastically different 2D maps of unheated control and salt samples are due to regulation of a proteolytic factor, rather than bon a fide proteome differences within the organism.

We also screened a protease inhibitor panel and, in agreement with previous reports (Frohlich et al., 2009) found that no inhibitor was capable of preventing the observed degradation in control samples (Fig. 2). All salinity samples remained intact with clean banding patterns irrespective of inhibitors (data not shown). In all cases, the non-heated samples remained degraded regardless of inhibitor used, and heating always prevented degradation. Identical patterns were obtained for samples that were either immediately electrophoresed following homogenization (Fig. 2) or allowed six hours incubation at $25^{\circ} \mathrm{C}$ prior to electrophoresis (data not shown), confirming that degradation did not occur until gel runs began. This observation is consistent with other reports of proteolytic problems arising during electrophoresis when proteases remain active and substrates become vulnerable due to unfolding (North and Beynon, 2001).

Although the protease inhibitor panel tested indicated that neither any individual inhibitor nor cocktail was capable of controlling degradation, it is impossible to conclude that the observed degradation is therefore not due to protease activity. Given our methodological focus on gel electrophoresis, we used 1D SDS-PAGE gels as our assay for degradation. Because degradation is occurring in denaturing conditions, it is possible that a reversible inhibitor would fail to effectively interact with the responsible protease(s) under these chemical conditions. The additional possibility of multiple proteases acting, each requiring a different type of inhibitor, should also be considered as samples are comprised of wholeorganism homogenate. The use of a phenol:chloroform extraction procedure should have removed nucleic acids from the samples, eliminating this class of molecules from consideration (Antonioli et al., 2009). If a protein-based molecule is responsible for the observed degradation, as seems likely, then ultimately one or more proteases are likely being regulated by salinity exposure. Our data do indicate that negative regulation is occurring, rather than positive regulation of a direct inhibitor, but it is presently impossible to determine if this is direct or the result of an indirect cascade involving various other proteins as well. Exploration of the specific mechanism awaits further study.

Until examining the "preserved" gels created via salinity treatment or heating, the full extent of proteome degradation occurring in "control" samples of D. pulex was difficult to comprehend. The evidence for high constitutive proteolysis in Daphnia extracts prepared by conventional methods should be taken into careful consideration in any study of the Daphnia proteome and our findings are not limited in usefulness to 2DGE, as any method which relies solely upon chemically denaturing conditions (such as various LC-MS/MS protocols) to control proteolysis will prove inadequate. It should be noted that in a LC-MS/MS study 
which utilized excised bands from a 1D gel, Frohlich et al. observed that $34 \%$ of proteins were identified from more than one band and speculated that this could have been caused by either in vivo posttranscriptional, in vivo posttranslational, or in vitro post-homogenization proteolysis (Frohlich et al., 2009). Our data indicate that no post-homogenization proteolysis occurs until samples are exposed to denaturing conditions and suggest that the observed diversity of molecular masses for any given protein is likely due to pre-existing in vivo processing, as long as proper care has been taken to avoid artifactual proteolysis.

\section{Conclusion}

Our data suggest that there exist in D. pulex one or more proteolytic factors with the following characteristics: 1) endogenously active within the organism under normal control conditions, 2) negatively regulated in vivo during salinity stress, 3) biochemically stable under typically denaturing conditions and 4) inactivated in vitro by heat.

\section{Acknowledgments}

Nellie Wilcox and Candice Young for Daphnia culture maintenance, Jim Clegg and Ben Jarrett for helpful commentary, Celia Chen for providing strains and valuable advice, and two anonymous reviewers for their highly useful suggestions. The project described was supported by a UC-Davis Floyd and Mary Schwall Fellowship in Genetics, UC-Davis Jastro-Shields Fellowship, NSF grant IOS- IOS-1049780, and NIH Award Number P42ES004699-25 from the National Institute of Environmental Health Sciences. The content is solely the responsibility of the authors and does not necessarily represent the official views of the National Institute of Environmental Health Sciences or the National Institutes of Health.

\section{Literature cited}

Antonioli P, Bachi A, Fasoli E, Righetti PG. Efficient removal of DNA from proteomic samples prior to two-dimensional map analysis. J Chromatogr A. 2009; 1216:3606-3612. [PubMed: 19081104]

Bianchini A, Wood CM. Sodium uptake in different life stages of crustaceans: The water flea daphnia magna strauss. J Exp Biol. 2008; 211:539-547. [PubMed: 18245630]

Colbourne JK, Pfrender ME, Gilbert D, Thomas WK, Tucker A, Oakley TH, Tokishita S, Aerts A, Arnold GJ, Basu MK, Bauer DJ, Caceres CE, Carmel L, Casola C, Choi JH, Detter JC, Dong Q, Dusheyko S, Eads BD, Frohlich T, Geiler-Samerotte KA, Gerlach D, Hatcher P, Jogdeo S, Krijgsveld J, Kriventseva EV, Kültz D, Laforsch C, Lindquist E, Lopez J, Manak JR, Muller J, Pangilinan J, Patwardhan RP, Pitluck S, Pritham EJ, Rechtsteiner A, Rho M, Rogozin IB, Sakarya O, Salamov A, Schaack S, Shapiro H, Shiga Y, Skalitzky C, Smith Z, Souvorov A, Sung W, Tang Z, Tsuchiya D, Tu H, Vos H, Wang M, Wolf YI, Yamagata H, Yamada T, Ye Y, Shaw JR, Andrews J, Crease TJ, Tang H, Lucas SM, Robertson HM, Bork P, Koonin EV, Zdobnov EM, Grigoriev IV, Lynch M, Boore JL. The ecoresponsive genome of daphnia pulex. Science. 2011; 331:555-561. [PubMed: 21292972]

Dowd WW, Renshaw GM, Cech JJ Jr, Kültz D. Compensatory proteome adjustments imply tissuespecific structural and metabolic reorganization following episodic hypoxia or anoxia in the epaulette shark (hemiscyllium ocellatum). Physiol Genomics. 2010; 42:93-114. [PubMed: 20371547]

Frohlich T, Arnold GJ, Fritsch R, Mayr T, Laforsch C. Lc-ms/ms-based proteome profiling in daphnia pulex and daphnia longicephala: The daphnia pulex genome database as a key for high throughput proteomics in daphnia. BMC Genomics. 2009; 10:171. [PubMed: 19383153]

Kurien BT, Scofield RH. Common artifacts and mistakes made in electrophoresis. Methods Mol Biol. 2012; 869:633-640. [PubMed: 22585529]

North, MJ.; Beynon, RJ. Prevention of unwanted proteolysis. In: Beynon, R.; Bond, JS., editors. Proteolytic enzymes. United States: Oxford University Press; 2001. p. 211-232.

Refardt D, Ebert D. Inference of parasite local adaptation using two different fitness components. J Evol Biol. 2007; 20:921-929. [PubMed: 17465903] 
Schwerin S, Zeis B, Lamkemeyer T, Paul RJ, Koch M, Madlung J, Fladerer C, Pirow R. Acclimatory responses of the daphnia pulex proteome to environmental changes. Ii. Chronic exposure to different temperatures (10 and 20 degrees c) mainly affects protein metabolism. BMC Physiol. 2009; 9:8. [PubMed: 19383147]

Zeis B, Lamkemeyer T, Paul RJ, Nunes F, Schwerin S, Koch M, Schutz W, Madlung J, Fladerer C, Pirow R. Acclimatory responses of the daphnia pulex proteome to environmental changes. I. Chronic exposure to hypoxia affects the oxygen transport system and carbohydrate metabolism. BMC Physiol. 2009; 9:7. [PubMed: 19383146] 


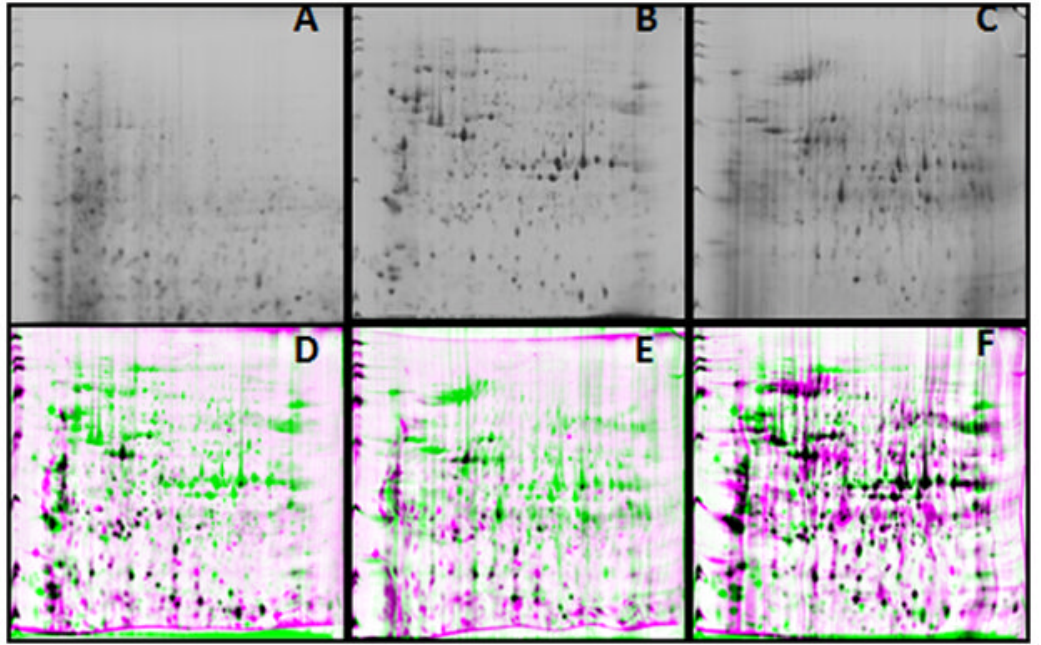

Figure 1.

Individual images and paired comparisons of representative two-dimensional gels prepared using pooled whole-organism samples from the following treatments: 12 hour control (15mOsm), 12 hour in vivo sodium chloride exposure (150mOsm), and control with in vitro post-homogenization sample heating. Both salinity exposure and heating result in less degradation compared to 'control' samples, as seen by a greater number of high molecular weight spots, greater coverage of the $2 \mathrm{D}$ space, and a lack of low molecular weight breakdown products and "smear" (lower left of gels). Note that of all comparisons, only the heated and salt gels appear to align; control samples are largely non-overlapping with any other treatment. Key: A) control B) in vivo salt-treatment C) heated control D) overlay of control (pink) vs salt (green) E) control (pink) vs heated control (green) F) salt (green) vs heated control (pink). 

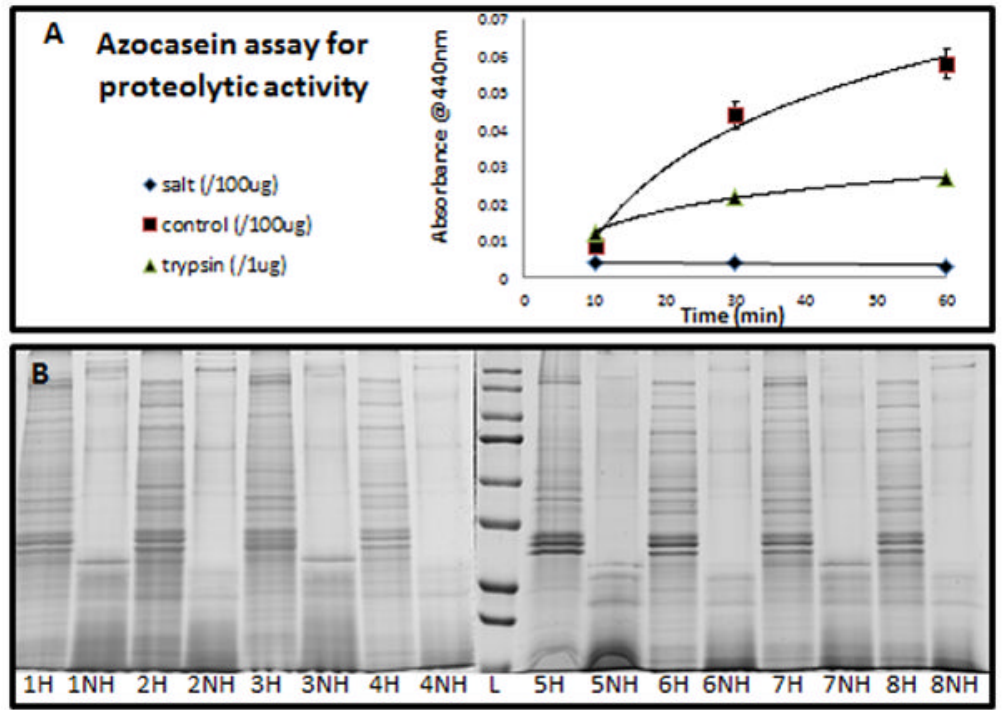

Figure 2.

A) Proteolytic activity rates were assessed using an azocasein assay. Absorbance values (+/standard error) at 440nm were measured for reactions terminated after 10, 30, or 60 minutes. B) Samples were homogenized in RIPA buffer containing the following protease inhibitors: 1) E-64 2) EST(E64-D) 3) Leupeptin 4) Pepstatin A 5) TLCK, HCL 6) TPCK 7) Complete Minitab 8) no inhibitor. Degradation was visually assesed using 1D SDS-PAGE. Samples were either heated at $95^{\circ} \mathrm{C}(\mathrm{H})$ or not heated $(\mathrm{NH})$ prior to loading to allow degradation to occur during electrophoresis. No protease inhibitor tested was capable of preventing visible degradation and separate samples left at room temperature for six hours had identical degradation patterns to those represented, confirming that degradation did not occur at the homogenization step but only after introduction of denaturing conditions. 

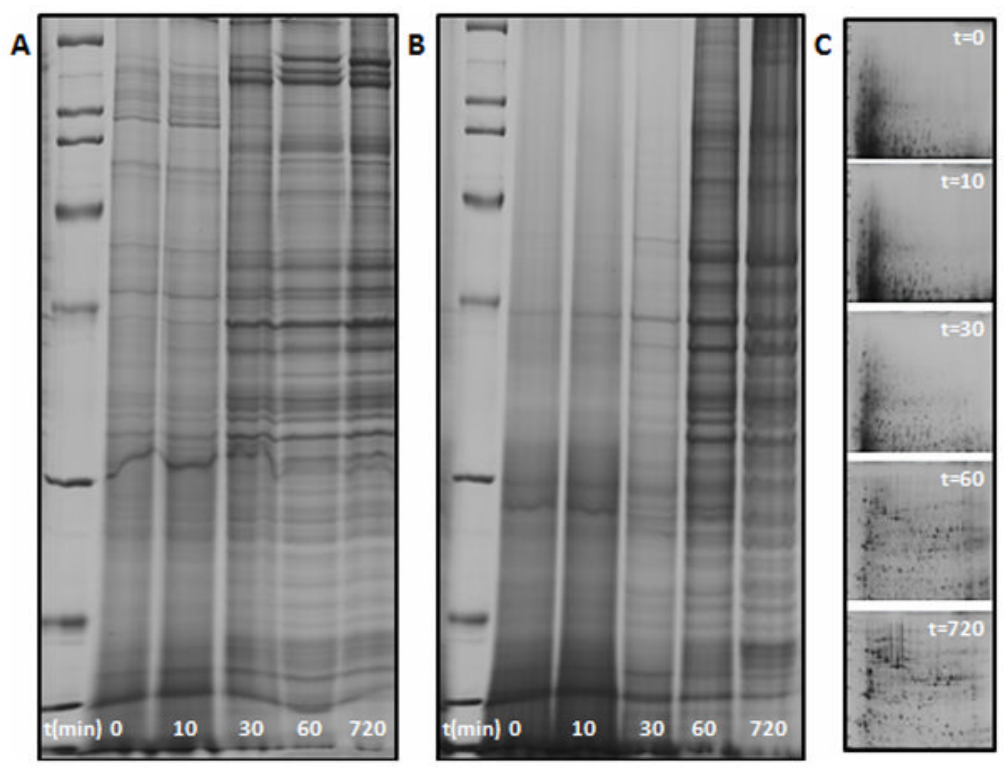

Figure 3.

1D SDS-PAGE gels of homogenized $D$. pulex samples in either A) RIPA homogenization buffer or B) high-urea UT buffer. Samples were taken at the following time points following exposure to $150 \mathrm{mOsm}$ media: $0 \mathrm{~min}$ (pre-exposure control), $10 \mathrm{~min}, 30 \mathrm{~min}, 60 \mathrm{~min}, 720$ min (12hr). For samples in RIPA, intact high MW bands are visible in all timepoints; these bands are lost with a corresponding increase in low MW smear in control/early timepoint samples once processing into high-urea buffer has occurred. This degradation effect does not occur in samples with in vivo salinity exposure of at least 60 minutes. 2D gels from each timepoint also displayed $(\mathbf{C})$. 


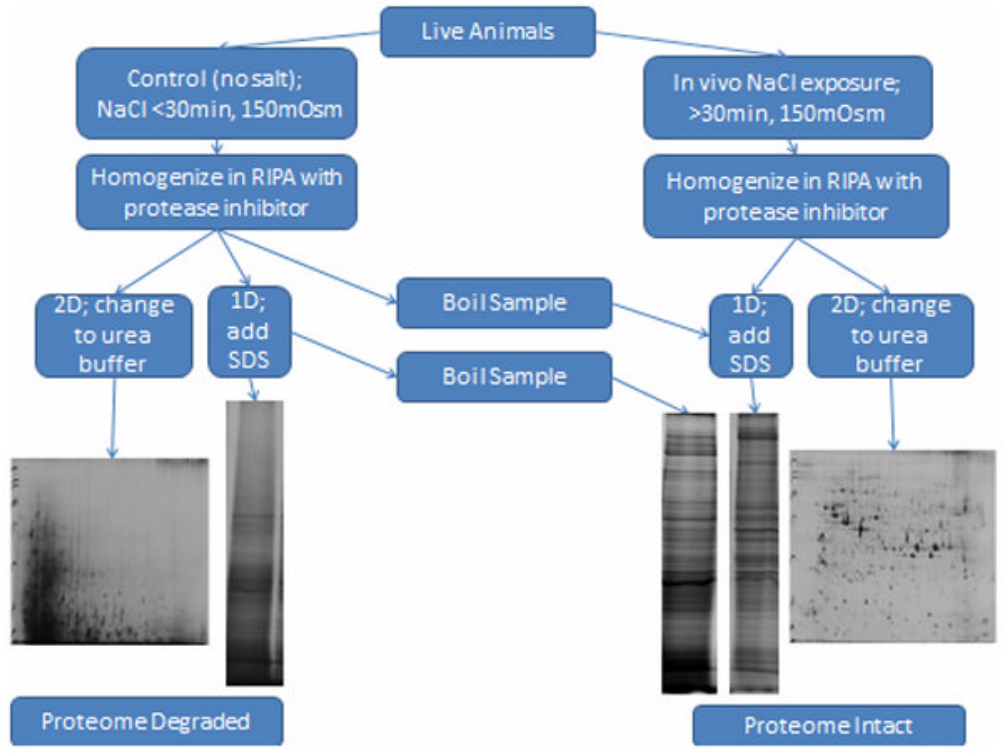

Figure 4.

Flowchart summary of experimental procedure variables capable of controlling proteomic degradation, with representative gel images. 


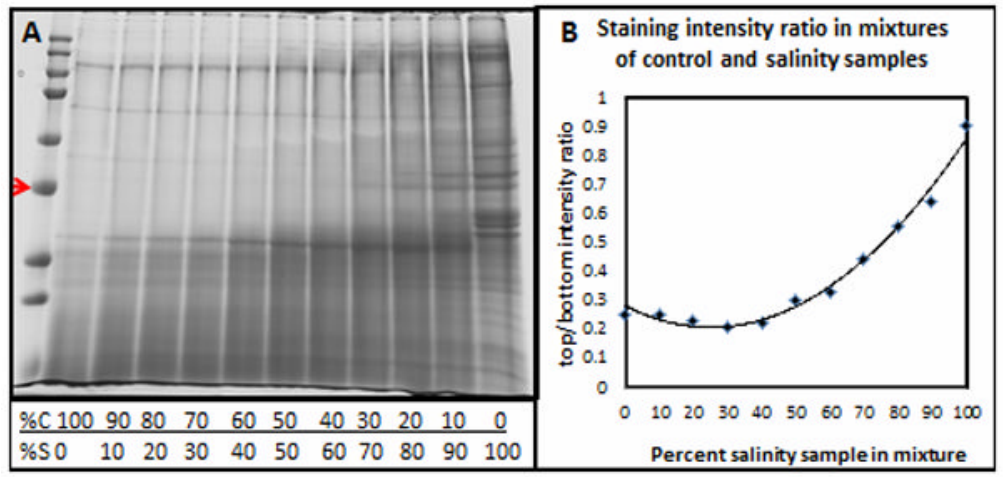

Figure 5.

A) Native 1D SDS-PAGE of preparations with varying proportions of control (C) and salinity (S) sample mixed together prior to loading. Samples were not heated prior to loading to allow degradation to occur. B) A ratio of staining intensity above and below the $37 \mathrm{kD}$

MW marker (arrow, A) was used to quantify the relative amount of degradation occurring in mixtures in $\mathrm{A}$, where a higher ratio indicates less degradation. These ratio data were plotted against the percentage of salinity sample in the total mixture. An exponential equation generated the best fit line $\left(y=0.0001 x^{2}-0.0059 x+0.2835 ; R^{2}=0.9842\right)$. Posthomogenization mixing of samples indicates that degrading activity is transferred from control (C) to salinity (S) samples, rather than salinity samples transferring inhibition to control samples. 